\title{
FINITE BOREL MEASURES ON SPACES OF CARDINALITY LESS THAN $c$
}

\author{
R. J. GARDNER AND G. GRUENHAGE
}

\begin{abstract}
Let $\kappa<c$ be uncountable. We prove, among other results, that every $\alpha$-realcompact space of cardinality $\kappa$ is Borel measure-compact if and only if there is a set of reals of cardinality $\kappa$ whose Lebesgue measure is not zero.
\end{abstract}

1. Introduction. Which diffused finite Borel measures exist on 'small' topological spaces, that is, spaces whose cardinality is less than $c$, the power of the continuum? Before attempting to answer this question, the authors knew two relevant results. Firstly, when the space is not Borel-complete (see $\$ 2$ for all definitions), there does exist a nontrivial diffused finite Borel measure. This is an atomic measure; the best known example is Dieudonné's measure on the ordinals less than or equal to $\omega_{1}$. It is a 'real' measure, in that no axioms other than those of ZFC (Zermelo-Fraenkel set theory together with the axiom of choice) are needed for its existence. Secondly, Martin's axiom implies that the Lebesgue measure of any set of reals of cardinality less than $c$ is zero. This follows from a result of Martin and Solovay [10, p. 167]; the same proof shows that there are no nontrivial finite Borel measures on any separable metric space of cardinality less than $c$.

In this paper, we first show that the existence of a nonatomic measure on a set of cardinality $\kappa, \kappa<c$, depends entirely on the existence of a set of reals of cardinality $\kappa$ whose Lebesgue measure is not zero. In fact, this is rather easily proved using standard techniques. It follows that Martin and Solovay's result using Martin's axiom extends not only to separable metric spaces, but to any Borel-complete space of cardinality less than $c$; under Martin's axiom, this includes all hereditarily paracompact, and indeed hereditarily weakly $\theta$-refinable spaces (see [4]). (If we insist that the measures be regular, we may drop the word "hereditarily".)

From the result mentioned above, it follows that if every set of reals of cardinality $\kappa, \kappa<c$, has Lebesgue measure zero, then every $\alpha$-realcompact (resp., Borel-complete) space of cardinality $\kappa$ is Borel measure-compact (resp., Borel measure-complete). In $\S 4$, we show that the converse is true.

2. Definitions. Suppose $(X, \Sigma, \mu)$ is a measure space. An atom is a set $A \in \Sigma$ such that $\mu(A)>0$, and whenever $B \in \Sigma$ and $B \subset A$, then $\mu(B)=\mu(A)$ or $\mu(B)=0$.

Received by the editors January 14, 1980 and, in revised form, August 5, 1980; presented to the Society, April 25, 1980 at Davis, California.

1980 Mathematics Subject Classification. Primary 54D20, 54G20, 54H99; Secondary 28A30, 28 A35.

Key words and phrases. Borel measure, regular measure, Borel-complete space, measure-compact space, $\alpha$-real compact space, Martin's axiom. 
The measure $\mu$ is said to be nonatomic if $\mu$ has no atoms. A Borel measure is a measure defined on the Borel subsets of a topological space $X$.

A finite Borel measure $\mu$ is regular if $\mu(B)=\sup \{\mu(F): F \subseteq B, F$ is closed $\}$, for each Borel set $B$, and diffused if $\mu(\{x\})=0$ for each point $x$. A space is Borel-complete [5] (respectively, $\alpha$-realcompact [2]) if each ultrafilter of Borel (respectively, closed) sets with the countable intersection property is fixed. Equivalently, $X$ is Borel-complete if for every two-valued Borel measure $\mu$ which is locally zero on an open set $G \subseteq X$, we have $\mu(G)=0$; and $X$ is $\alpha$-realcompact if every two-valued, locally zero, regular Borel measure on $X$ is identically zero. If we omit 'two-valued' from these alternative definitions we define Borel measure-complete and Borel measure-compact.

Suppose $\kappa$ is an uncountable ordinal not cofinal with $\omega$. Dieudonnés measure $\nu$ on $[0, \kappa)$ is defined as follows. A set $E \subseteq[0, \kappa)$ has $\nu$-measure 1 if and only if $E$ contains an unbounded, closed set. It is not hard to prove that $\nu$ is a Borel measure (see [6, p. 231, Example 10]).

\section{Nonatomic measures.}

Proposition 3.1. Suppose $\kappa$ is a cardinal less than $c$. Then the following are equivalent:

(a) Every set of reals of cardinality $\kappa$ has Lebesgue measure zero.

(b) Every nontrivial finite measure space $(X, \Sigma, \mu)$ with $|X|=\kappa$ has an atom.

(c) If $(X, \Sigma, \mu)$ is a nontrivial finite measure space with $|X|=\kappa$, then $\mu$ has the form $\mu(E)=\Sigma_{n} \mu\left(E \cap A_{n}\right)$ for each $E \in \Sigma$, where $\left(A_{n}\right)$ is a disjoint collection of atoms.

Proof. That (c) $\Rightarrow$ (b) is trivial, and (b) $\Rightarrow$ (c) follows from standard arguments. If $X$ is a set of reals of cardinality $\kappa$ with positive outer measure, then Lebesgue measure restricted to $X$ is nonatomic. Thus (b) $\Rightarrow(a)$.

It remains to prove (a) $\Rightarrow(b)$. We will show instead that the negation of (b) implies there is a subset of the Cantor set $2^{\omega}$ of cardinality $\kappa$ whose Haar measure is not zero. Suppose $(X, \Sigma, \mu)$ is a finite nonatomic measure space with $|X|=\kappa$. We may assume $\mu(X)=1$. A nonatomic measure takes on all values in $[0, \mu(X)]$ (see $[11,(18-28)]$ ). Thus, for each finite sequence $f$ of 0 's and l's (we view $f$ as a function from $n$ into 2 for some integer $n$ ), we can construct $A(f) \in \Sigma$ such that

(i) $A(\varnothing)=X$;

(ii) $A(f)=A\left(f^{\wedge} 0\right) A\left(f^{\wedge} 1\right)$, where " $f^{\wedge} i$ " means " $f$ followed by $i$ ";

(iii) $A\left(f^{\wedge} 0\right) \cap A\left(f^{\wedge} 1\right)=\varnothing$; and

(iv) $\mu(A(f))=2^{-|f|}$.

Here, $\varnothing$ is the empty sequence, and $|f|$ denotes the length of the sequence $f$.

Now let $C=\left\{f \in 2^{\omega}: \bigcap_{n \in \omega} A\left(\left.f\right|_{n}\right) \neq \varnothing\right\}$. Suppose the Haar measure of $C$ is zero. Let $U$ be an open set containing $C$ such that the Haar measure of $U$ is less than 1. There is a cover of $C$ by a disjoint collection $\mathscr{B}$ of open sets of the form $B(g)=\left\{f \in 2^{\omega}: g \subset f\right\}$, where $g: n \rightarrow\{0,1\}$ for some $n \in \omega$. Let $\Sigma^{\prime}=\{A(g)$ : $B(g) \in \mathscr{B}\}$. Then $\Sigma^{\prime}$ covers $X$ and $\mu\left(\cup \Sigma^{\prime}\right)<1$ (since $\mu(A(g))$ is the Haar measure of $B(g)$ ), which is a contradiction. Thus $C$ has positive outer measure. 
REMARK. The method of proof above is used, for example, in Maharam's theorem [9] and in some basic results on measurable cardinals (see, for example [1, Chapter 6]). In fact, Proposition 3.1 can be obtained from Maharam's theorem (we thank Professor D. Stone for outlining this in a letter to us), but the direct argument above is considerably shorter.

Since we are interested in Borel measures, we state the following corollary.

COROllary 3.2. Suppose $\kappa$ is a cardinal less than $c$. Then the following are equivalent:

(a) Every set of reals of cardinality $\kappa$ has Lebesgue measure zero.

(b) Every nontrivial finite (regular) Borel measure on a space of cardinality $\kappa$ has a (closed) atom.

(c) Every nontrivial finite (regular) Borel measure $\mu$ on a space of cardinality $\alpha$ is of the form $\mu(E)=\Sigma_{n} \mu\left(E \cap B_{n}\right)$ for each Borel set $E$, where $\left(B_{n}\right)$ is a disjoint collection of (closed) atoms.

4. Measure-compactness and measure-completeness. In this section we consider Borel measure-compactness vis-à-vis $\alpha$-realcompactness, and Borel measure-completeness vis-à-vis Borel-completeness. For example, suppose we ask whether every $\alpha$-realcompact space $X$ of cardinality $\kappa<c$ is Borel measure-compact; i.e., if every regular, locally zero, 2-valued Borel measure on $X$ is trivial can one conclude that every regular, locally zero, finite Borel measure on $X$ is trivial? From Proposition 3.1 , it follows that the answer is affirmative if each subset of $\mathbf{R}$ of cardinality $\boldsymbol{\kappa}$ has Lebesgue measure zero. On the other hand, if $\kappa$ is a real-valued measurable cardinal, then the discrete space of cardinality $\kappa$ is a counterexample. It turns out we can still get a (nonmetrizable) counterexample with only the assumption that there exists a subset of $\mathbf{R}$ of cardinality $\kappa$ which does not have Lebesgue measure zero.

Proposition 4.1. Suppose $\kappa$ is a cardinal less than $c$. Then the following are equivalent:

(a) Every set of reals of cardinality $\kappa$ has Lebesgue measure zero.

(b) Every Borel-complete space of cardinality $\kappa$ is Borel measure-complete.

(c) Every $\alpha$-realcompact space of cardinality $\kappa$ is Borél measure-compact.

Proof. That (a) $\Rightarrow$ (b) and (a) $\Rightarrow$ (c) follows from Proposition 3.1.

We show $(b) \Rightarrow(a)$ and $(c) \Rightarrow(a)$. Suppose there is a set $Y$ of reals of cardinality $\kappa$ which has positive outer measure. Let $\kappa_{0}$ be the least such cardinal. Note that $\kappa_{0}$ is not cofinal with $\omega$. We show there is a space of cardinality $\kappa_{0}$, and hence $\kappa$, which is Borel-complete (and so $\alpha$-realcompact) but not Borel measure-compact (and so not Borel measure-complete). We use a construction due to $\mathbf{R}$. Haydon (see [7], or [4, Example 3.6]). Let $Y_{0} \subseteq \mathrm{R}$ such that $\left|Y_{0}\right|=\kappa_{0}$ and $Y_{0}$ has positive outer Lebesgue measure. Let $\phi$ be a 1-1 map from $Y_{0}$ onto $\left[0, \kappa_{0}\right)$ and let $X=\{(\alpha, t)$ : $\alpha<\phi(t)\}$. By Haydon's arguments, $X$ is Borel-complete. The product measure $\nu \times \lambda$, where $\nu$ is Dieudonné's measure on $\left[0, \kappa_{0}\right)($ see $\$ 2$ ) and $\lambda$ is Lebesgue 
measure, when restricted to $X$, is a nontrivial, diffused, regular, finite Borel measure which is locally zero; and so $X$ is not Borel measure-compact.

The following result on Baire measures can be obtained in exactly the same way. We refer the reader to [4] and [7] for additional information on measure-compact spaces.

Proposition 4.2. Suppose $\kappa$ is a cardinal less than $c$. The following are equivalent:

(a) Every set of reals of cardinality $\kappa$ has Lebesgue measure zero.

(b) Every nontrivial finite Baire measure on a space of cardinality $\kappa$ has an atom.

(c) Every realcompact space of cardinality $\kappa$ is measure-compact.

A topological space $X$ is a Radon space if for every finite Borel measure $\mu$ on $X$, we have $\mu(B)=\sup \{\mu(K): K \subset B, K$ compact $\}$ for each Borel set $B$. We conclude this section with the following question.

Question 4.3. Is every compact Hausdorff Borel-complete space of cardinality less than $c$ a Radon space?

It follows from known results that this question is equivalent to the question of whether every compact Hausdorff Borel-complete space is Borel measure-complete. Thus, by Proposition 4.1, the answer is affimative if every set of reals of cardinality less than $c$ has Lebesgue measure zero. On the other hand, if $\kappa$ is a real-valued measurable cardinal, then the one-point compactification of a discrete space of cardinality $\kappa$ is a counterexample. But we do not know if it is possible to construct a counterexample without assuming the existence of a real-valued measurable cardinal.

5. Remarks. As we noted in the introduction, if Martin's axiom holds, then each set of reals of cardinality $\kappa<c$ has Lebesgue measure zero. (In fact, Martin and Solovay prove that a stronger statement holds: the union of less than $c$ sets of Lebesgue measure zero is also of Lebesgue measure zero. However, they also remark that there are models of set theory such that each set of reals of cardinality $\kappa<c$ has Lebesgue measure zero, but $[0,1]$ is the union of $\aleph_{1}$ sets of measure zero.) On the other hand it is pointed out in [10] that there are models of set theory in which there is a set of reals of cardinality $\kappa<c$ which is not of Lebesgue measure zero.

One consequence of (c) of Proposition 4.1 is that if $\kappa<c$, then $\kappa$ is not real-valued measurable. Kunen [8] has proved that if each set of reals of cardinality $\kappa<c$ has Lebesgue measure zero, for all $\kappa<c$, then $\kappa<c$ is not real-valued measurable.

We mention one related result. Fremlin [3] has shown, using Martin's axiom, that if $\mu$ is a Radon measure, then the union of less than $c$ sets of $\mu$-measure zero has inner $\mu$-measure zero.

Finally, note that (b) (resp., (c)) of Proposition 4.1 can be stated in the stronger form: every diffused (regular) finite Borel measure on a Borel-complete (resp., $\alpha$-realcompact) space of cardinality less than $c$ is identically zero. 


\section{REFERENCES}

1. F. R. Drake, Set theory, North-Holland, London, 1974.

2. N. Dykes, Generalizations of realcompact spaces, Pacific J. Math. 33 (1970), 571-581.

3. D. H. Fremlin, Uncountable powers of $\mathbf{R}$ can be almost Lindelöf, Manuscripta Math. 22 (1977), 77-85.

4. R. J. Gardner, The regularity of Borel measures and Borel measure-compactness, Proc. London Math. Soc. (3) 30 (1975), 95-113.

5. A. W. Hager, G. D. Reynolds and M. D. Rice, Borel-complete topological spaces, Fund. Math. 75 (1972), 135-143.

6. P. R. Halmos, Measure theory, Van Nostrand, Princeton, N. J., 1950.

7. R. Haydon, On compactness in spaces of measures and measure-compact spaces, Proc. London Math. Soc. (3) 29 (1974), 1-16.

8. K. Kunen, Inaccessibility properties of cardinals, Doctoral Dissertation, Stanford Univ., 1968.

9. D. Maharam, On homogeneous measure algebras, Proc. Nat. Acad. Sci. U.S.A. 28 (1942), 108-111.

10. D. A. Martin and R. M. Solovay, Internal Cohen extensions, Ann. Math. Logic 2 (1970), 143-178.

11. W. Pfeffer, Integrals and measures, Dekker, New York, 1977.

Department of Mathematics, Auburn University, Auburn, Alabama 36830 (Curtent address of G. Gruenhage)

Current address (R. J. Gardner): Department of Mathematics, North Texas State University, Denton, Texas 76205 